\title{
A Method for Sex Prediction from Transfer Stains
}

\author{
Nabanita Basu' ${ }^{1}$ and Samir Kumar Bandyopadhyay ${ }^{2 *}$ \\ ${ }^{1}$ Advisor to Chancellor, JIS University, India \\ ${ }^{2}$ Department of Computer Science and Engineering, JIS University, India
}

*Corresponding author: Samir Kumar Bandyopadhyay, Department of Computer Science and Engineering, JIS University, India

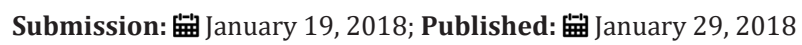

\begin{abstract}
Violent criminal activities under most circumstances are accompanied by large spillage of blood. A bloodstain formed due to accumulation of liquid blood on an absorbent surface such as carpet, fabric, clothing, bed sheet, curtains etc. is commonly referred to as 'Saturation Stain'. This paper proposed a computer based system that could effectively predict the gender of an individual using SVM basic kernel functions.
\end{abstract}

Keywords: Support vector machine; Kernel; Facial features; Gender identification; Crime scene

\section{Introduction}

Gender is among the strongest predictors of crime, particularly violent crime. Arrest, self-report, and victimization data consistently show that men and boys commit significantly more crime, both serious and not, than women and girls. This pattern persists despite data indicating that crimes committed by females may be rising. Evidence also suggests that males are generally more aggressive than females, even before the preschool years.

The results of the Biosocial Study confirmed past research which had demonstrated gender differences in the prevalence of crime. Males engaged in more crime and violence than females, and they were more likely to repeat their crimes. However, the Biosocial Study also corresponded with some research and theory which had indicated gender differences in the prediction of crime. With some exceptions, biological factors were found to be more predictive of crime among females, whereas environmental factors were found to be more predictive of crime among males. More factors overall were correlated with crime among females than males.

Gender differences in the prediction of crime may substantially affect differences in prevalence. Gender becomes particularly important when assessing the "criminal careers" 80 of individual offenders, because predictive influences may vary depending on an individual's age and physiological development. An analysis of a criminal career focuses on two key elements:

a. Participation-the difference between those who do or do not commit a crime,

b. Frequency-the number of crimes an active offender commits.
81 Certain factors, such as the offender's age at the initiation of a criminal career, the escalation and desistance of the offender's criminal behavior, and the policy approaches for restraining criminal careers, are influential in determining the onset and continuation of criminal careers. 'Gender-based violence' and 'violence against women' are terms that are often used interchangeably as most gender-based violence is inflicted by men on women and girls. However, it is important to retain the 'gender-based' aspect of the concept as this highlights the fact that violence against women is an expression of power inequalities between women and men. A multidisciplinary approach to crime is crucial for understanding both why crime occurs and the conditions for any possible gender differences. In crime investigation this chapter suggests that classification of gender plays an important role for understanding behavioral pattern of crime.

Over the period of time, automated classification of gender has gained enormous significance and has become an active area of research. Many researchers have put a lot of effort and have produced quality research in this area. Still, there is an immense potential in this field because of its utility in many areas like monitoring, surveillance, commercial profiling and human-computer interaction. Security applications have utmost importance in this area. Gender classification can be used as part of a face recognition process.

Gender classification can be stated as inferring female or male from a collection of facial images. It is actually binary classification, there exist different methods for gender classification, such as gait, iris, hand shape and hair, yet the prominent methods to achieve the goal are based on facial features. Gender identity is defined 
as a personal conception of oneself as male or female (or rarely, both or neither). This concept is intimately related to the concept of gender role, which is defined as the outward manifestations of personality that reflect the gender identity. Gender identity, in nearly all instances, is self-identified, as a result of a combination of inherent and extrinsic or environmental factors, gender role, on the other hand, is manifested within society by observable factors such as behavior and appearance. The basic idea in this work was to develop a computer based system that could effectively predict the gender of an individual.

\section{Literature Reviews}

Gender recognition is an interesting problem that can be used to boost the performance of several important applications such as face recognition and video surveillance. Gender classification can be utilized as an indexing technique to reduce the search space for automatic and manual recognition techniques. Further, other areas such as human computer interaction also have many interesting applications ranging from automatically identifying gender of individuals to image search over the internet.

Gender classification problem is an active research area which has attracted a great deal of attention recently. It is a challenging pattern recognition problem. Generally gender classification involves a process of determining the gender of a subject from face images. The face images analysis plays an important role in computer vision. Face images analysis has been successfully used in many applications ranging from biometric to robotic-human interaction. Current face detection applications operate with high accuracy as compared to gender classifications systems, because gender classification systems do not offer same level of performance and accuracy. Face is a very important biometric feature of human. Automatic recognizing and analyzing of face is one of challenging tasks in object recognition. Successful performing this task allows many applications in human computer interaction.

It is desirable to find features that can be used to determine people's gender. In general, it is believed that male and female have unique physical characteristics, such as fingerprint, palmprint, and face. Even in $[1,2]$ writers reported that male and female shows the difference in the way they talk or walk. When gender can be detected based on their physical characteristics, unknown criminal or victims of natural disasters can be identified faster. As one of the biometrics means, the fingerprint commonly classified into five major classes, namely whorl, left-loop, right-loop, arch and tented arch. It is advantageous when fingerprints further classified based on the gender of its owner. Furthermore, when the gender of the fingerprint is known then extracting and matching modules can be developed properly [3]. Many researchers have taken a close look to find fingerprint features that can be used to classify gender. They commonly determine ridge related features [4,5], analyze frequency domain [6], and implement 2D Discrete Wavelet Transforms [7]. Gender plays a significant role in our interactions in society and with computers [8]. Actually Gender classification is a binary classification problem in which one has to predict an image belongs to a man or woman. It is an easy job for a person but a challenging one for computers [9].

\section{Proposed Method}

In this paper, novel methodologies have been proposed to achieve the goal of gender classification. Input image set are preprocessed to perform noise removal, histogram equalization, size normalization and then face detection is performed. Secondly, Feature Extraction from facial image is performed. Finally to evaluate the performance of the proposed algorithm, experiments have been performed on various image set that contain equal proportion of male and female by using suitable binary SVM classifier which will classify the data set into two categories i.e. male or female. The basic idea behind this paper is to appraise the performance of SVM basic kernel functions which has been employed to detect and classify the human gender into two class problem i.e.

a. Male class and

b. Female class.

These functions read the feature(s) of human facial image(s) as input. The proposed algorithm is then executed on the elementary features of a human facial image i.e. eyes, nose, lips and their all possible combinations. Finally based on the accuracy percentage of the computed result the admissible outcomes of the Kernel Functions have been realized.

Cross Validation of the Shuffled Matrix is one of the key steps I $n$ Gender Recognition Algorithm. It resolves the issues like over fitting of images. Besides if the original data set is appropriately cross validated, it can be effortlessly divided into the train set and the test set. The size of the train set and the test set however depends on the degree of the cross validation technique. Like the 'hold out technique divides the original set into two equal sized sets, while the other techniques like ' 10 fold' cross validation and ' 5 fold 'cross validation dissociates the primitive data into 10 segments and 5 segments respectively. Each time one segment is tested to predict the class labels of the undetermined set after acquiring the result of the training of the remaining (n-1) segments.

Studies have consistently shown higher rates of offending for males than for females, and especially higher rates of violence. Gender differences in the development of social cognition may help to explain gender differences in crime and violence. How an individual ultimately responds to a stressful life event or risk factor depends on how that event is perceived, which, in turn, depends on an individual's cognitive processes. Social information-processing skills allow individuals to encode information, interpret and consider risks and benefits of a particular action, and determine an appropriate response based on their repertoire of behavioral scripts. It is not necessarily suggested that deficiencies in cognitive capabilities cause crime, but rather that certain ways of processing social information and certain social cognitive memory structures help to protect the individual from personal, social, environmental, or situational pressures towards criminal behavior. 
Transfer stains are common stain patterns at a crime scene. Commendable work has been done towards locating and enhancing bloodstain patterns on difficult surfaces. Difficult surfaces in this context include dark colored surfaces, human skin, fabrics etc. Weapons have steep geometric edges. In lieu of circumstantial evidence, it is often straightforward to identify the weapon that had particularly left the imprint. However, prediction of gender from bloody broken prints left at crime scene is way more complicated and difficult to analyze. While fingerprints have been vastly dealt with, work relating to prediction of gender of an individual from broken bloody footprint impressions stands limited. To mend this loophole a dataset was created to document the bloody footprints of footprints of individuals (Figure 1).

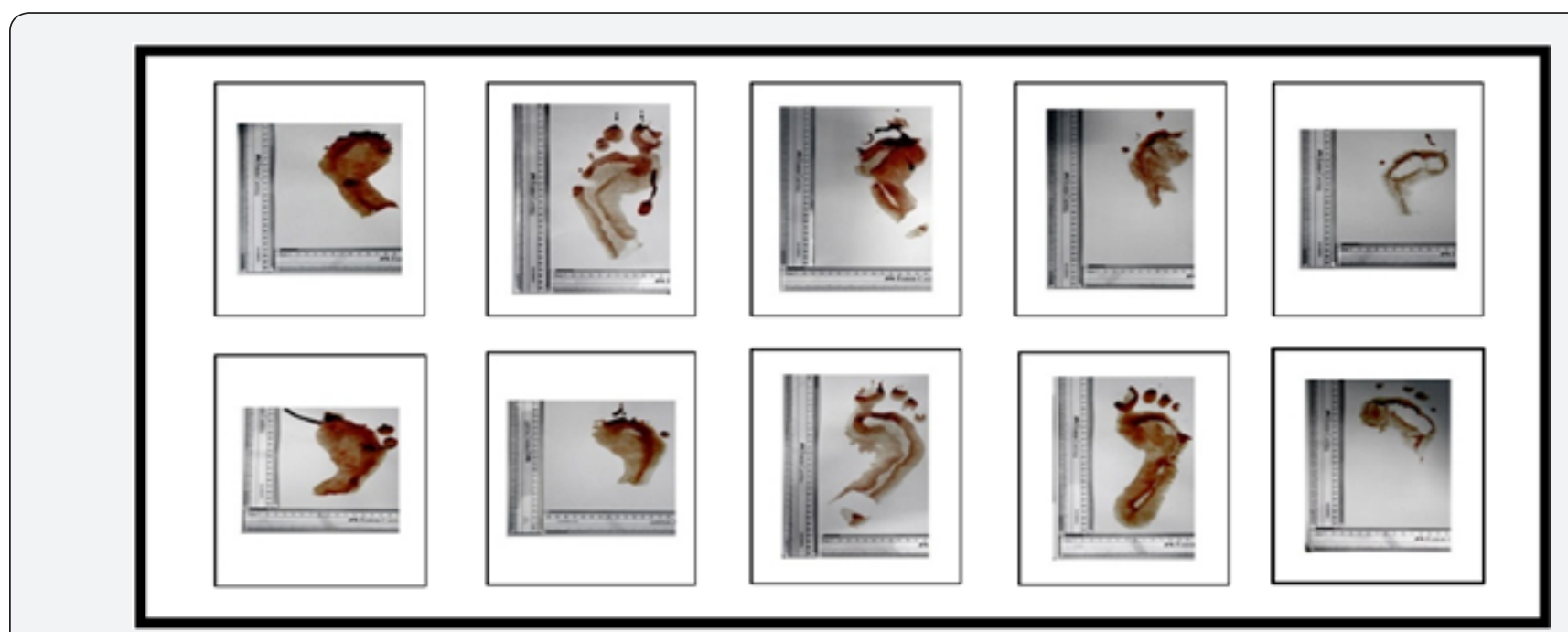

Figure 1: Snapshot of the Footprint database that has been developed. The aspect ratio for each image was maintained when mounting the images on a canvas, so that each image in the database could be of the same dimension $\left(250 \times 400 \mathrm{pixel}^{2}\right)$.

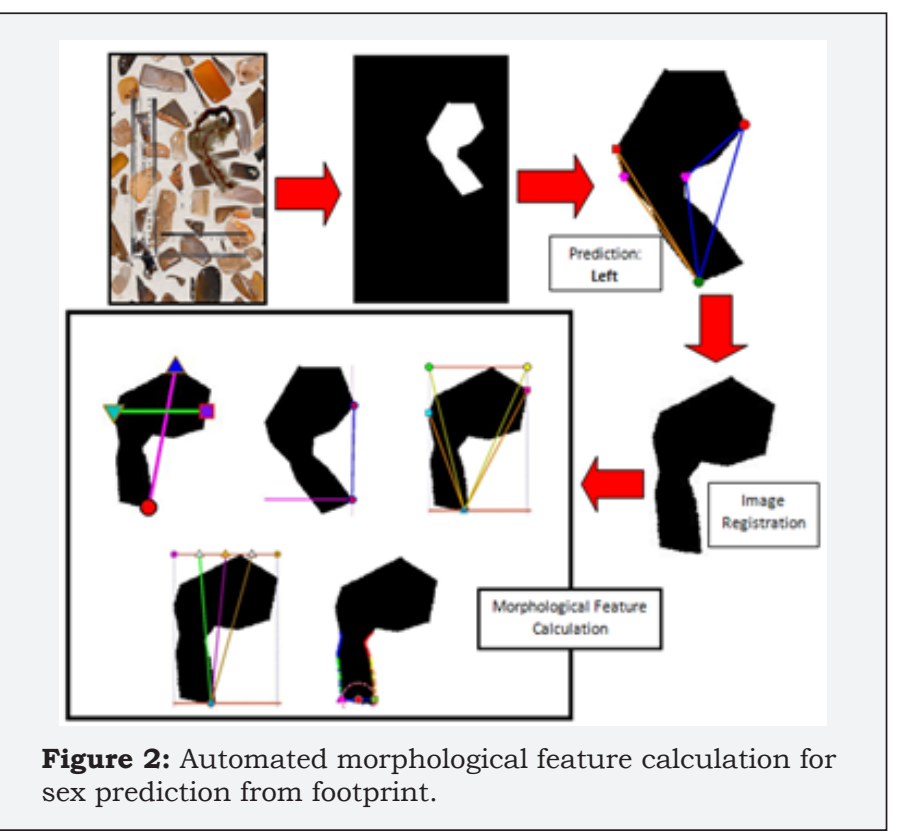

As a precursor, human subjects who consented to be part of the project were asked to step on blood pool and thereby walk on herbarium sheet plank without shoe. Five men and five women (i.e total of 10 individuals) in the age group of 18 to 65 years consented to be a part of the experiment. Data collected was curated. Supervised learning techniques were used to predict gender of an individual from a broken bloody footprint/shoeprint irrespective of the color, texture of the target surface on which it was recorded. The features used for sex classification were length of footprint transfer stain, breadth , angle of walking, aspect ratio of stain, length calculation based on closest possible geometric approximation of the stain pattern, breadth approximation, length of approximated digit ends from the approximated tip of foot, heel area etc. It is shown in Figure 2. An automated wrapper feature based sex prediction system provided a prediction accuracy of 0.8347 (Sensitivity - 0.8361, Specificity -0.8333) for the 121 data point strong footprint database while a fully automated filter feature based prediction system achieved a prediction accuracy of 0.8017(Sensitivity- 0.8033, Specificity-0.8000). Figure 3 provides an overview of the data model that was developed. Given that only morphological features are used by the system, other broken foot patterns (namely, muddy footprint, dirt print etc.) can also be used by the system for sex prediction.

However, in developing the software only plain, smooth, nonabsorbent target surfaces were considered for recording of stain patterns. So on these terms the workings of the software is limited to predicting gender only from a broken footprint stain formed on a plain, smooth, non-absorbent target surface.

A closer look at the aforementioned methodologies developed shall highlight that both the systems cannot work on prints developed on Fabric as also on other porous /absorbent, rough target surface. In particular fabric stains are difficult to analyze owing to the flexibility of fabric target surface, large variance in the weave, permeability etc. 


\section{Conclusion}

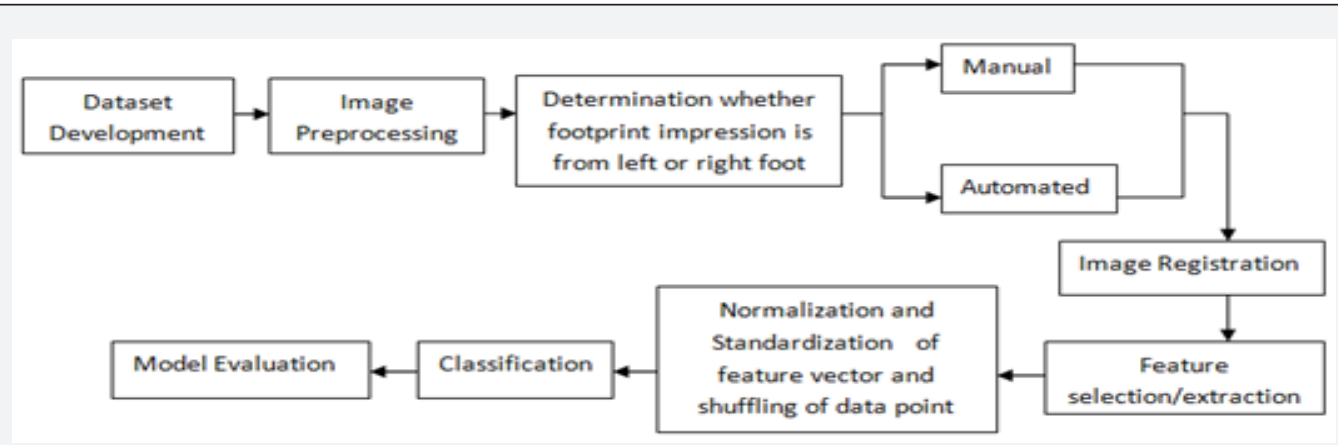

Figure 3: Data model developed for gender estimation from bloody broken crime scene footprint/shoeprint images [Manual refers to label based separation of left foot sole print from the right footprint].

A fully automated system has been proposed for foot print ROI (Region of Interest) extraction, subsequent to which automatic separation of left and right foot occurs. This is closely followed by automated morphological feature extraction and subsequent classification of the sex of the foot sole owner into either of the 2 categories, male or female. The wrapper feature based model recorded an accuracy of 0.8347 (Sensitivity -0.8361 , Specificity-0.8333) on the 121 strong dataset that had been subjected to leave-one-out cross validation. The filter feature based model was documented to record an accuracy of 0.8017(Sensitivity-0.8033, Specificity-0.8000) over the same dataset. A semi-automated module was prepared to support user based ROI extraction from textured surface or surfaces that do not mimic the background that has been used for recording footprints. Given that the model uses only foot morphology features for sex prediction, the model can also be extended to predict gender from mud foot sole print, dirt foot sole print etc.

\section{References}

1. Arai k, Asmara RA (2012) Human gait gender classification in spatial and temporal reasoning. Int J Adv Res Artificial Intelligence 1(6): 1-6.

2. Arai $K$, Asmara RA (2014) Human gait gender classification using 3D discrete wavelet transform feature extraction 3(2): 12-17.
3. Frick M, Modi SK, Elliott SJ, Kukula EP (2008) Impact of gender on fingerprint recognition. $5^{\text {th }}$ international conference on information technology and applications 717-721.

4. Nayak VC, Rastogi P, Kanchan T, Yoganarasimha K, Kumar GP, et al. (2010) Sex differences from fingerprint ridge density in Chinese and Malaysian population. Forensic Sci Int 197(1-3): 67-69.

5. Midiora EO, Ojo O, Yekini N, Tubi T (2012) Analysis, design and implementation of human fingerprint patterns system towards age \& gender determination, ridge thickness to valley thickness ratio (rtvtr) \& ridge count on gender detection. Int J Adv Res Artificial Intelligence 1(2): 5763.

6. Kaur R, Mazumdar SG (2012) Fingerprint based gender identification using frequency domain analysis. IJAET 3(1): 295-299.

7. Tom RJ, Arulkumaran T (2013) Fingerprint based gender classification using 2D discrete wavelet transforms and principal component analysis. Int J Eng Trends Technol 4(2): 199-203.

8. Nazir M, Ishtiaq M, Batool A, Jaffar MA, Mirza AM (2016) Feature selection for efficient gender classification recent advances in neural networks, fuzzy systems \& evolutionary computing ISBN: 978-960-474195-3.

9. Khaung Tin HH (2012) Perceived Gender Classification from Face Images. I J Modern Education and Computer Science 4(1): 12-18. 